\title{
SEMIALGEBRAIC BOREL-MOORE-HOMOLOGY
}

\author{
HANS DELFS
}

Dedicated to the memory of Gus Efroymson

Borel and Moore developed an homology theory for locally compact topological spaces using sheaf theoretical methods ([2], see also [3]). It allows us to consider not only homology with compact support (which is the same as classical singular homology) but also homology with arbitrary support. This general concept of homology has important applications. A striking example is the proof that every algebraic variety over $\mathbf{R}$ (resp. over $\mathbf{C}$ ) has a fundamental class [1]. To obtain similar results for varieties over an arbitrary real closed field $R$, or over its algebraic closure $C=R(\sqrt{-1})$, we are forced to introduce a semialgebraic version of Borel-Moore-homology. In this paper we will sketch how this may be done. (In [4] and [6] we handled the special case of complete support). Semialgebraic Borel-Moore-homology may even be of interest in the case $R=\mathbf{R}$, because it is far simpler and more elementary than the classical sheaf theoretical theory.

$R$ denotes a real closed field. Let $M$ be an affine semialgebraic space over $R$ [5] and $A$ be a semialgebraic subset of $M$. We can triangulate $M$, $A$ simultaneously, i.e., there exists a finite geometric simplicial complex $X$ over $R$, a subcomplex $Y$ of $X$ and a semialgebraic isomorphism $\varphi: X \approx$ $M$ which maps $Y$ onto $A[6, \S 2]$. Notice that the simplicial complex $X$ is not complete in general. Some faces may be missing. Adding these missing faces we obtain a simplicial complex $\bar{X}$, the closure of $X . \bar{X}$ is a simplicial complex in the classical sense and a complete semialgebraic space.

The basic building blocks in classical algebraic topology are closed simplices. But not all simplices occuring in $X$ are closed. Nevertheless, they seem to contribute to the homology of $M$. So it is quite natural to take the open simplices as the building blocks of a homology theory for semialgebraic spaces. This idea will be formalized in the following.

Definition 1. An abstract simplicial complex $K$ is a pair $(E(K), S(K))$ consisting of a set $E(K)$, called the vertices of $K$, and a set $S(K)$ of nonempty finite subsets of $E(K)$, called the simplices of $K$, such that $E(K)=$ $\bigcup(s \mid s \in S(K)) . K$ is called closed if, in addition, every non-empty subset $t$ of a simplex $s \in S(K)$ is also a simplex. 
In the literature only closed abstract complexes are considered (cf., e.g., [7, 3.1]). Adding all missing faces of $K$ to $S(K)$ gives us a closed abstract complex $\bar{K}$, the closure of $K$.

We can associate an abstract simplicial complex $K(X)=(E(X), S(X))$ to $X$ as follows; $E(X)$ is the set of vertices of $\bar{X}$; a subset $s$ of $E(X)$ belongs to $S(X)$ if and only if the elements of $s$ span an open simplex of $X$.

We assume from now on that the given semialgebraic space $M$ is locally complete. This means that every point of $M$ has a complete semialgebraic neighborhood $[5, \S 9]$ and corresponds to the topological notion "locally compact". (The space $V(R)$ of $R$-rational points is locally complete for every $R$-variety $V$ ). Then $X$ is open in its closure $\bar{X}$. It also means that the abstract complex $K(X)$ is an open subcomplex of its closure $\overline{K(X)}=$ $K(\bar{X})$ (i.e., $\overline{K(X)}-K(X)$ is a closed subcomplex of $\overline{K(X)})$. For every abstract complex $K$ with this property we can form the oriented chain complex

$$
C_{*}(K, G):=C_{*}(\bar{K}, G) / C_{*}(\bar{K}-K, G),
$$

defined as the quotient of the oriented chain complexes [7, 4.1] of the closed simplicial complexes $\bar{K}, \bar{K}-K$ ( $G$ an abelian group).

REMARK. The generators of $C_{n}(K(X), G)$ are in one-to-one correspondance with the open $n$-simplices of the geometric complex $X$. For any open $n$-simplex $\sigma$ the boundary $\partial \sigma$ is the alternate sum of all oriented open $(n-1)$-dimensional faces of $\sigma$ which belong to $X$, i.e., $\partial \sigma$ is obtained from the classical boundary chain just by omitting all faces which are not contained in $X$.

Definition 2. $H_{n}(M, G):=H_{n}\left(C_{*}(K(X), G)\right)$ is called the $n$-th homology group of $M$ with closed support and coefficients in $G$.

The homology groups of $M$, defined in [4] and [6] as the homology groups of the largest subcomplex $K(X)_{0}$ of $K(X)$ which is closed, are now more precisely called the homology groups with complete support and denoted by $H_{n}^{c}(M, G)$.

Notice that the choice of a triangulation $\varphi: X \neg M$ means, among other things, that we have chosen a "completion" $\bar{M}$ of $M$, namely $\bar{M}=$ $\bar{X}$. By definition, $H_{n}(M, G)=H_{n}^{c}(\bar{M}, \bar{M}-M, G)$. The latter group does not depend on the triangulation of $\bar{M}, M$ [4]. But, since different triangulations lead, in general, to different completions of $M$, it is a non trivial question whether $H_{n}(M, G)$ is independent on the triangulation of $M$. This is indeed true and can be proved using the homotopy axiom for semialgebraic cohomology with complete support.

Homology with closed support is functorial under proper semialgebraic maps. It has all the properties of Borel-Moore-homology. This can be 
verified using easy "simplicial arguments". In the case $R=\mathbf{R}$ it coincides with Borel-Moore-homology.

Let $U$ be an open semialgebraic subset of $M$ and $A=M \backslash U$. There is a canonical restriction map

$$
H_{*}(M, G) \stackrel{r}{\longrightarrow} H_{*}(U, G) .
$$

If we triangulate $M$ and $U$ simultaneously, then $r$ is induced by "forgetting" the simplices outside of $U$. A short look at the simplices proves the following proposition.

Proposition 1. There is a canonical exact sequence

$$
\cdots \rightarrow H_{n}(A, G) \rightarrow H_{n}(M, G) \stackrel{r}{\longrightarrow} H_{n}(U, G) \rightarrow H_{n-1}(A, G) \rightarrow \cdots .
$$

Assume now that $M$ is a connected $n$-dimensional semialgebraic manifold over $R$.

Proposition 2. $H_{n}(M, \mathbf{Z} / 2)=\mathbf{Z} / 2 \cdot z \cong \mathbf{Z} / 2$.

Here, $z$ is the cycle represented by the sum of $n$-simplices of $M$. For $M$ orientable, Proposition 2 is also true with $\mathbf{Z} / 2$ replaced by $\mathbf{Z}$.

THeOREM 3. (see [1] for $R=\mathbf{R}$, [6] for the complete case). Let $V$ be an $n$-dimensional algebraic variety over $R$. Then the space $V(R)$ of real points of $V$ has a fundamental class mod 2, i.e., there exists a (uniquely determined) homology class $z \in H_{n}(V(R), \mathbf{Z} / 2)$ whose restriction $z \mid M$ to every component $M$ of $V_{\text {reg }}(R)$ generates $H_{n}(M, \mathbf{Z} / 2)$.

The proof is easy. We immediately reduce to the case $V$ irreducible. Let $U:=V_{\text {reg }}(R)$ and $A:=V_{\text {sing }}(R)=V(R) \backslash U$. If $\operatorname{dim} V_{\text {sing }} \leqq n-2$, then the exact sequence in Proposition 1 shows that $H_{n}(V(R), \mathrm{Z} / 2) \simeq$ $H_{n}(U, \mathbf{Z} / 2)$. In general, let $\pi: V^{\prime} \rightarrow V$ be the normalization of $V$ and $z^{\prime}$ be the fundamental class of $V^{\prime}(R) . \pi_{R}=\pi \mid V^{\prime}(R): V^{\prime}(R) \rightarrow V(R)$ is proper and the image $z$ of $z^{\prime}$ under

$$
\left(\pi_{R}\right)_{*}: H_{n}\left(V^{\prime}(R), \mathbf{Z} / 2\right) \rightarrow H_{n}(V(R), \mathbf{Z} / 2)
$$

is the fundamental class of $V(R)$.

If $V$ is an $n$-dimensional variety over the algebraic closure $C=R(\sqrt{-1})$ of $R$, connected in the Zariski-topology, then similar arguments show that $H_{2 n}(V(C), \mathbf{Z})$ is isomorphic to $\mathbf{Z}$. It posesses a canonical generator $z$, the fundamental class, corresponding to the natural orientation of $V_{r e g}(C)$. Using a refinement of the classical simplicial proof of Poincaré-duality (with compact support) we can prove Poincaré-duality for semialgebraic manifolds over $R$ and arbitrary support, without use of spectral sequences. Applying this result to $V_{\text {reg }}(C)$ considered as a semialgebraic space over 
$R$, we then can define the intersection multiplicities of algebraic cycles on a variety over any algebraically closed field of characteristic zero in a purely topological (i.e., semialgebraic) way (see [1] for the case $C=\mathbf{C}$ ).

\section{REFERENCES}

1. A. Borel, A. Haefliger, La classe d'homologie fondamentale d'un espace analytique, Bull. Soc. Math. France 89 (1961), 461-513.

2. - J. C. Moore, Homology theory for locally compact spaces, Mich. Math. J. 7 (1960), 137-159.

3. G. Bredon, Sheaf Theory, McGraw Hill, New York, 1967.

4. H. Delfs, Kohomologie affiner semialgebraischer Räume, Diss., Univ. Regensburg, 1980.

5. - M. Knebusch, Semialgebraic topology over a real closed field II: Basic theory of semialgebraic spaces, Math. Z. 178 (1981), 175-213.

6. - On the homology of algebraic varieties over real closed fields, J. reine angew. Math. 335 (1982), 122-163.

7. E. Spanier, Algebraic Topology, McGraw Hill, New York, 1966.

FAKUltät Für MATHEMATIK DER UNIVERSITÄT REgenSBURG, D-8400 REgenSBURG, F.R.G. 